\title{
Large Scale Convection in Stars : Towards a Model for the Action of Coherent Structures
}

\author{
Michel Rieutord ${ }^{1,2}$ and Jean-Paul Zahn ${ }^{1}$ \\ ${ }^{1}$ Observatoire Midi Pyrenees, 14 av. E. Belin, 31400 Toulouse, France \\ ${ }^{2}$ CERFACS, 42 av. Coriolis, 31057 Toulouse, France
}

\begin{abstract}
We show that, representing the descending fluid in a convection zone by a porous medium, the differential rotation of the (rising) fluid is very close to that in an axisymmetric madel of the convection zone with anisotropic viscosity.
\end{abstract}

\section{Introduction}

The computation of large scale convective flows in astrophysical objects is certainly one of the great challenge of contemporary astrophysics. The computation of such flows is a basic requirement for understanding the dynamical behaviour of stars or planets. The huge Reynolds number of the flow, due to the large size of the objects, makes the flow highly turbulent and so very difficult to compute. The main problem is that we do not have, for the moment, a complete theory of turbulence.

Until now two approaches have been considered: firstly the mean field approach and secondly direct numerical simulations. These two approaches possesses some major drawbacks which need to be recapitulated.

The mean field approach (see Rüdiger, 1989 and references therein) is concerned, in the case of stars, with the determination of the long term evolution of the mean axisymmetric fields. Indeed, we wish to know the nature of the mechanism that maintains the differential rotation or governs the magnetic activity. The mean is thus defined as to smooth out longitudinal and short-time dependence of the phenomena. For instance, if we are interested in the velocity field, we set :

$$
\boldsymbol{u}=\boldsymbol{U}+\boldsymbol{u}^{\prime},
$$

where $\boldsymbol{u}^{\prime}$ stands for the fluctuation around the mean $\boldsymbol{U}=\langle\boldsymbol{u}\rangle$. The evolution of the mean $U$ can be derived, and, in the case of an incompressible fluid, gives the Reynolds equation

$$
\partial_{t} U_{i}+U_{j} \nabla_{j} U_{i}=-\nabla_{i} P+\nu \Delta U_{i}-\partial_{j} R_{i j}
$$


where $R_{i j}=\left\langle u_{i}^{\prime} u_{j}^{\prime}\right\rangle$ is the Reynolds stress tensor. This last quantity carnot be computed unless we have a theory of turbulence that tells us the relation between this tensor and $\boldsymbol{U}$. In the case where separation of scales applies (i.e. $\left|\boldsymbol{u}^{\prime}\right| \ll|\boldsymbol{U}|$ ), then the equation for $\boldsymbol{u}^{\prime}$ can be made linear and solved for some given forcing. An expression for $R_{i j}$ is then obtained. The main question is then about the validity of such an approximation. Indeed, it is questionable if this approximation can be applied to large scale non-axisymmetric modes. This is the main drawback of the method.

The second approach, the numerical one, is still concerned with solving (2), but now the mean is taken on a much smaller scale: essentially it is a smoothing of all the small scales not resolved by the numerical scheme. Here, as above, the relation between the Reynolds stress tensor and the mean velocity field is not known. However, such relation may be easier to obtain, since we are now dealing with small scales which have certainly been derived and nothing proves to be satisfactory in all cases. Let us recall a few existing (subgrid) models.

First, Smagorinski's model (1963) where :

$$
R_{i j}=-C_{S} \Delta^{2} \sqrt{S_{i j} S^{i j}} S_{i j}
$$

with $S_{i j}=\left(\partial_{i} U_{j}+\partial_{j} U_{i}\right)$. This model has been tested against direct numerical simulations and turned out to be able to represent only $16 \%$ of the true $R_{i j}$ (Fertziger, 1985). We can also mention the $K \varepsilon$ model (see Peyret and Taylor, 1983), the renormalization group approach (Yakhot and Orszag, 1986) and a new version of Smagorinski's approach by Leith (1989). The main drawback with these models is their lack of predictability: either because, like the $K \varepsilon$ model, they have constants that need adjusting or, like Smagorinski's, they lack universality, leaving their predictions questionable.

\section{The role of coherent structures?}

Coherent structures (we refer here mainly to vortex tubes) play an important role which remains to be understood. It is well known that their presence reflects the nongaussianity of the turbulence statistics which creates all the difficulties of the statistical approaches. One of the difficulties of modelling the action of such objects is due to their "two-scaledness", that is to say that such an object as a vortex tube has a length of the order of the integral scale whilst it has a width of a few dissipation scales (see for instance Vincent and Meneguzzi, 1990). In large eddy simulations, such objects would appear as singularities of the flow since their thickness is not resolved while their length is. We may also note, from the spectral point of view, that such structures constitute a direct link between small and large scales and thus allow a direct exchange of energy.

We shall model these structures in a very crude way. We assume that they are purely passive. First we make them solid and we fix them in space (no advection by the flow). They thus make a porous medium through which the fluid passes. 
To see the first consequences of such an assumption we computed the differential rotation of the solar convection zone (SCZ) when it is modeled by such a medium. We note that a porous medium was also used by Bretherton and Spiegel (1968) to model the SCZ for studying the solar spin-down. titlea3.The solar convection zone as a porous medium? Let us make the following assumptions : the SCZ is a porous medium where the solid part stands for the descending fluid (the plumes) and the fluid saturating this porous medium stands for the rising fluid; it is injected at the base of the SCZ and extracted at the top (which is our boundary conditions on the flow).

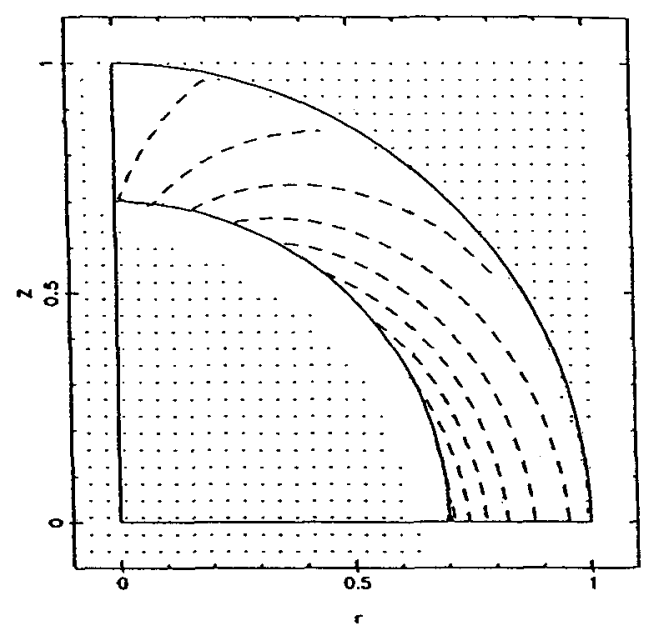

Fig. 1. Contours of constant angular velocity obtained with the porous medium model. The scaling factor $\mathrm{E}=\nu / 2 \Omega k_{\theta}=10$. The shape of the contours is only slightly modified when $k_{r} / k_{\theta}$ or $\mathrm{E}$ are changed.

We shall assume in addition that the fluid is incompressible and that the motion is steady and axisymmetric. Such flow obeys the following equations:

$$
\left\{\begin{aligned}
2 \Omega \times v & =-\nabla P+\nu[k]^{-1} v, \\
\operatorname{div} v & =0
\end{aligned}\right.
$$

where $\nu[k]^{-1} v$ is the Darcy force characteristic of porous media (see Cushman $1990) .[k]$ is the permeability tensor which we take in the form

$$
\left[\begin{array}{ccc}
k_{r} & 0 & 0 \\
0 & k_{\theta} & 0 \\
0 & 0 & k_{\phi}
\end{array}\right]
$$

The anisotropy of the medium is then characterized by the ratio $k_{r} / k_{\theta}\left(k_{\theta}=k_{\phi}\right)$ which we take to 10: the medium is more permeable in the vertical direction than in the horizontal one. The resulting differential rotation is plotted on Fig. 1. The shape of the contours only slightly depends on the anisotropy. In Fig. 2 we 


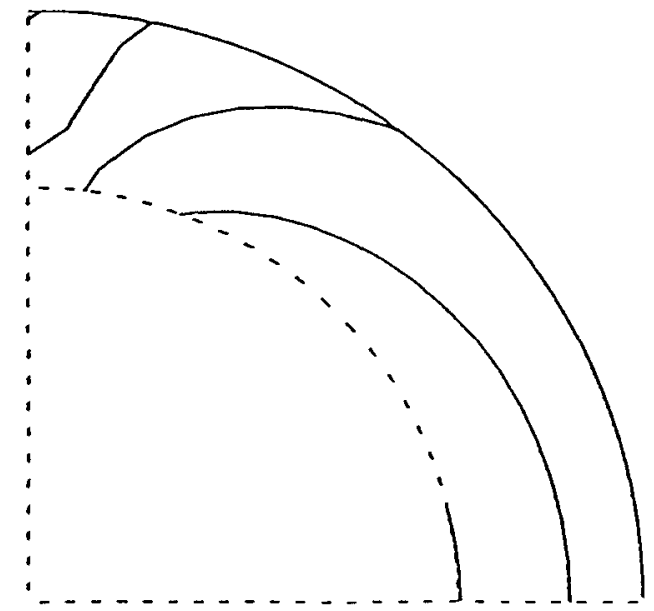

Fig. 2. Contours of constant angular velocity obtained with a numerical integration of anelastic axisymmetric equations of motion, using an anisotropic viscosity (20\% of anisotropy).

The anisotropy of the medium is then characterized by the ratio $k_{r} / k_{\theta}\left(k_{\theta}=k_{\phi}\right)$ which we take to 10 : the medium is more permeable in the vertical direction than in the horizontal one. The resulting differential rotation is plotted on Fig. 1. The shape of the contours only slightly depends on the anisotropy. In Fig. 2 we plotted the differential rotation obtained by Brandenburg and Rieutord (1990) using an axisymmetric, anelastic model with an anisotropic viscous tensor (the horizontal viscosity being larger than the vertical one). The two calculations give a similar distribution of angular velocity with a positive gradient $(\partial \Omega / \partial r>0)$. In both models the Coriolis force is the main contributor to the distribution, but the remarkable fact is that the model presented here is extremely simple. The next steps in this direction of investigation will include relaxing the restriction to a solid porous medium (coherent structures should be advected by the flow), computing their statistics, and allowing the structures to interact as a source of field.

\section{References}

Brandenburg, A., Rieutord, M.: 1990, in preparation.

Bretherton, F., Spiegel, E.A.: 1968, Astrophys. J. Lett. 153, L77

Cushman, A.: 1990, Dynamics of fluids in hierarchical porous media, Academic Press

Fertziger, J.: 1985, in Theoretical Approaches to Turbulence, eds. D.L. Dwoyer, M.Y. Hussaini, R.G. Voigt, Springer

Leith, C.: 1989, Phys. Fluids A 2, 297

Peyret, R., Taylor, T.D.: 1983, Computational Methods for Fluid Flow, Springer

Rüdiger, G.: 1989, Differential Rotation and Stellar Convection, Gordon and Breach, New York

Smagorinski, J.: 1963, Mon. Weather Rev. 91, 94

Vincent, A., Meneguzzi, M.: 1990, to appear in J. Fluid Mech.

Yakhot, V., Orszag, S.: 1986, J. Scientific Computing 1, 3 